\title{
Poder corneal poscirugía fotoablativa para el cálculo de la lente intraocular aportado por el Pentacam-AXL
}

\section{Corneal power after photoablative surgery for intraocular lens calculation using Pentacam-AXL}

\author{
Taimi Cárdenas-Díaz*, M. Teresa González-Hernández y Michel Guerra-Almaguer \\ Instituto Cubano de Oftalmología Ramón Pando Ferrer, La Habana, Cuba
}

\section{Resumen}

Objetivo: Comparar el poder de la lente intraocular calculada con la potencia refractiva total corneal (TRCP), las lecturas queratométricas efectivas (EKR) aportadas por el Holladay Report del topógrafo Pentacam-AXL y el poder corneal real (True K) utilizado por la fórmula de Barret True $K$ incorporada en el propio equipo, con los valores obtenidos por el método de historia clínica $(\mathrm{MHC})$ en pacientes con cirugía fotoablativa previa para corregir la miopía. Método: Se realizó un estudio transversal en 99 ojos de 52 pacientes miopes poscirugía fotoablativa en el Servicio de Cirugía Refractiva del Instituto Ramón Pando Ferrer, de noviembre de 2018 a noviembre de 2019. Se estudiaron características demográficas, refractivas y biométricas que incluyeron poderes corneales aportados por el Pentacam-AXL. Se compararon el poder corneal y el poder de la lente intraocular calculada. Resultados: La edad media fue de $25.71 \pm 4.46$, hubo predominio femenino (67.3\%), y el equivalente esférico fue de $-0,06 \pm 0,34$ dioptrías. Solo hubo diferencias significativas en la queratometría media $(p=0.02)$ $y$ el poder de la lente calculado con esta $(p<0.01)$ al compararlo con el obtenido por el MHC; no así con la EKR, la TRCP y el True K. Conclusiones: Las lecturas queratométricas efectivas que aporta el módulo Holladay Report del Pentacam, la potencia refractiva total corneal y el poder corneal real utilizado por la fórmula de Barret True K no difieren del poder corneal obtenido por el MHC en ojos con cirugía fotoablativa previa para corregir la miopía, por lo cual pueden emplearse en el cálculo de la lente intraocular.

Palabras clave: Poder corneal. Cirugía fotoablativa. Lente intraocular.

\section{Abstract}

Objective: To compare the intraocular lens power calculated with the total refractive corneal power (TRCP), the effective keratometric readings (EKR) provided by the Holladay Report of the Pentacam-AXL topographer and the real corneal power (True K) used by the Barret True $K$ formula incorporated in the equipment, with the values obtained by the clinical history method (CHM) in patients with previous photoablative surgery for myopia correction. Method: A cross-sectional study was carried out in 99 eyes of 52 myopic patients after photoablative surgery in the Refractive Surgery Department of the Ramón Pando Ferrer Institute from November 2018 to November 2019. Demographic, refractive and biometric characteristics were evaluated, including corneal powers obtained by the Pentacam-AXL. We compared corneal power and the intraocular lens power calculation. Results: Mean age was $25.71 \pm 4.46$ years, there was a female predominance $(67.3 \%)$ and the spherical equivalent was $-0.06 \pm 0.34$ diopters. There were only significant differences in mean keratometry $(p=0.02)$ and intraocular

Fecha de recepción: 11-05-2021 Fecha de aceptación: 07-07-2021 E-mail: taimicar@infomed.sld.cu DOI: 10.24875/RMO.M21000187
Disponible en internet: 07-09-2021 Rev Mex Oftalmol. 2021;95(6):245-251 www.rmo.com.mx 0187-4519/( 2021 Sociedad Mexicana de Oftalmología. Publicado por Permanyer. Este es un artículo open access bajo la licencia CC BY-NC-ND (http://creativecommons.org/licenses/by-nc-nd/4.0/). 
lens power calculated using keratometry $(p<0.01)$ compared with that calculated using the MHC; not so with EKR, TRCP and True K. Conclusions: The effective keratometric readings provided by the Holladay Report module of the Pentacam, the total corneal refractive power and the real corneal power used by the Barret True $K$ formula, do not differ from the corneal power obtained by the MHC in eyes with previous photoablative surgery for myopia correction, so they can be used for intraocular lens calculation.

Keywords: Corneal power. Photoablative surgery. Intraocular lens.

\section{Introducción}

Cada vez es más frecuente programar cirugías de cataratas en pacientes miopes a los que previamente se les ha efectuado cirugía refractiva corneal (CRC), la cual modifica la córnea central, por lo que los métodos convencionales de medición sobrestiman el poder corneal. La literatura actual sugiere que esta sobrestimación es de un $14-25 \%$ después de la cirugía refractiva'.

Durante los últimos 17 años se han desarrollado más de 30 fórmulas o métodos para el cálculo del poder de la lente intraocular (LIO), que pueden agruparse según la disponibilidad de datos: 1) cuando la queratometría o los cambios refractivos están disponibles (método de Seitz/Speicher), 2) cuando es posible recalcular el índice queratométrico (método de Savini, fórmula de Masket, fórmula de Barrett True-K, fórmula de regresión de Latkany, fórmulas (seis) de Awwad y método de historia clínica [MHC]) y 3) cuando no se dispone de la queratometría ni de los cambios refractivos (fórmulas PHL y Shammas-PL, método Maloney, fórmula Haigis-L, fórmula de Barrett True $K$ no histórica y fórmula de ópticas gaussianas) $)^{2,3}$.

El MHC ha sido bien conocido desde su introducción por Holladay en 1989 y Hoffer en 1995. Aunque se ha considerado como el método de referencia durante años, cada vez más estudios han indicado que es menos preciso que los nuevos métodos. Su exactitud depende de la disponibilidad de datos preoperatorios, y esto no siempre es posible. Los pacientes cambian de centro asistencial, en algunos no se anotan las queratometrías previas y otras veces el oftalmólogo que los atiende no es quien opera al paciente, y así solo se dispone del estado refractivo actual del paciente. Además, tiene una fiabilidad pobre cuando los datos son imprecisos ${ }^{2-4}$.

La necesidad de obtener el correcto poder corneal tras la cirugía fotoablativa para utilizarlo en el cálculo de la LIO es de interés creciente, ya que el número de pacientes con catarata y este antecedente cada día es mayor. Lo ideal para el cirujano es contar con un equipo que mida el poder corneal de manera directa y correcta, sin realizar cálculos ni inferencias. El topógrafo de Scheimpflug Pentacam-AXL ${ }^{5-10}$ constituye una herramienta muy útil en la estimación del poder corneal en pacientes con cirugía refractiva previa, debido a que tiene incorporado a su software aplicaciones para efectuar dicha mensuración, como la potencia refractiva total corneal (TRCP, total refractive corneal power), el poder corneal real (True $K$ ) empleado en la formula Barret True $K^{11,12}$ y el programa Holladay Report ${ }^{13}$. En los estudios realizados en el Instituto Cubano de Oftalmología Ramón Pando Ferrer (ICORPF) 3,14,15 para comparar los valores aportados por el topógrafo Pentacam con los del MHC en pacientes con CRC, se observó una mayor correlación con las lecturas queratométricas efectivas (EKR, Equivalent Keratometry Readings) a 3 y $4 \mathrm{~mm}$ en miopes, pero no se conoce con la TRCP y el True K. Por otra parte, en el propio ICORPF la fórmula Barret True $K$ incorporada al Pentacam-AXL resultó útil para el cálculo de la LIO en los pacientes con catarata y CRC previa ${ }^{16}$.

Ante estas alternativas se realizó el presente estudio con el objetivo de comparar el poder de la LIO calculado con la TRCP, las EKR aportadas por el Holladay Report del topógrafo Pentacam-AXL y el True $K$ utilizado por la fórmula de Barret True $K$ incorporada en el propio equipo, con los valores obtenidos por el MHC en pacientes con cirugía fotoablativa previa para corregir la miopía.

\section{Método}

Se trata de un estudio transversal en pacientes con cirugía fotoablativa previa para corregir la miopía realizada por un mismo cirujano en el servicio de cirugía refractiva del ICORPF, de noviembre de 2018 a noviembre de 2019. Se incluyeron los pacientes con 3 meses como mínimo desde la CRC por láser excímer y se excluyeron aquellos con afecciones y cirugías oculares previas.

Se estudiaron las características demográficas (sexo y edad), refractivas como el equivalente esférico y biométricas incluyendo la longitud axial y la profundidad 
de la cámara anterior, además de los poderes corneales aportados por el Pentacam-AXL ${ }^{5-10}$, ejemplos la queratometría media $(\mathrm{Km})$ medida dentro de los $3,00 \mathrm{~mm}$ centrales, así como la TRCP a los $4,00 \mathrm{~mm}$ que considera las condiciones reales ópticas de la córnea y las EKR del módulo Holladay Report a los $3,00 \mathrm{~mm}$, $4,00 \mathrm{~mm}$ y $4,50 \mathrm{~mm}$, que son las efectivas después de la CRC fotoablativa ${ }^{13-15}$. Se analizó también el poder corneal que utiliza el Pentacam-AXL para el cálculo de la lente con formula de Barret ${ }^{11,12,16}$, que denomina True $K$, y el calculado mediante el $\mathrm{MHC}^{2-4,14,15}$, que utiliza la fórmula $\mathrm{K}=$ Kpre + Rpre - Rpo, donde Kpre es el poder corneal preoperatorio, Rpre es la refracción preoperatoria y Rpo es la refracción posoperatoria. Este método, como bien se había planteado anteriormente, aún es

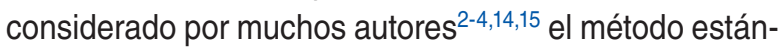
dar para obtener el poder corneal después de la CRC, por lo que fue usado como referencia en este estudio para establecer las comparaciones.

Se calculó el poder de la LIO con cada uno de estos poderes corneales. Para evitar el error de predicción de la posición efectiva de la lente (ELP, effective lens position) se empleó la fórmula de cuarta generación Haigis, que no emplea la queratometría como predictora de la ELP, para validar el resultado ${ }^{17}$. En el caso de las EKR del Holladay Report, la fórmula recomendada es la Holladay II, pero no está disponible en el Pentacam-AXL del ICORPF, y aunque sí lo está en el IOL Master 700, este último no permite introducir los valores de las EKR, y por eso se utiliza también la Haigis. En el caso de la formula Barret que viene incorporada en el Pentacam-AXL, de igual manera no dejó modificar el poder corneal y de ahí que se analizó el poder del lente dado por la misma y el poder corneal que utiliza (True K).

El análisis estadístico se realizó con la prueba $t$ para datos pareados, con una significación del $95 \%$. Una diferencia con un valor $p<0.05$ se consideró estadísticamente significativa.

El estudio cumplió con lo establecido en el Sistema Nacional de Salud y previsto en la Ley No. 41 de Salud Pública, en correspondencia con la Declaración de Helsinki ${ }^{18}$. Además, contó con la aprobación del comité de ética y científico de la institución de los autores, y todos los participantes firmaron el consentimiento informado.

\section{Resultados}

Se incluyeron 99 ojos de 52 pacientes con cirugía fotoablativa previa para corregir la miopía con un tiempo
Tabla 1. Características demográficas, refractivas y biométricas

\begin{tabular}{|c|c|c|}
\hline \multirow{2}{*}{$\begin{array}{l}\text { Sexo } \\
(n=52)\end{array}$} & Femenino, n (\%) & $35(67.3)$ \\
\hline & Masculino, n (\%) & $17(32.7)$ \\
\hline Edad (años) & $\begin{array}{l}\text { Media } \pm \mathrm{DE} \\
\text { (min-max) }\end{array}$ & $\begin{array}{l}25.71 \pm 4.46 \\
\quad(21-44)\end{array}$ \\
\hline $\begin{array}{l}\text { Equivalente esférico } \\
\text { (dioptrías) }\end{array}$ & $\begin{array}{l}\text { Media } \pm \mathrm{DE} \\
\text { (min-max) }\end{array}$ & $\begin{array}{l}-0.06 \pm 0.34 \\
(-1.75-0.50)\end{array}$ \\
\hline Longitud axial (mm) & Media $\pm \mathrm{DE}$ & $24.26 \pm 0.90$ \\
\hline $\begin{array}{l}\text { Profundidad de la } \\
\text { cámara anterior (mm) }\end{array}$ & Media $\pm \mathrm{DE}$ & $3.19 \pm 0.25$ \\
\hline
\end{tabular}

medio entre la CRC y el examen con Pentacam AXL de $6.30 \pm 3.56$ meses (rango: $3-12$ meses). Todos los pacientes fueron tratados mediante técnica de superficie LASEK (Laser Assisted Sub-Epithelial Keratomileusis).

En la tabla 1 se resumen las características demográficas, refractivas y biométricas de los pacientes. Hubo predominio del sexo femenino, representando un $67.3 \%$ del total de los pacientes. La edad promedio fue de $25.71 \pm 4.46$ años (rango: 21-44 años). El error refractivo promedio fue $-0.06 \pm 0.34$ dioptrías (D), lográndose el objetivo de la cirugía. La media de la longitud axial fue de $24.26 \pm 0.90 \mathrm{~mm}$ y la de la amplitud de la cámara anterior fue de $3.19 \pm 0.26 \mathrm{~mm}$ aportada por el Pentacam AXL.

En la tabla 2 se expone el poder corneal obtenido mediante el MHC y el topógrafo Pentacam AXL. El promedio del poder corneal con el MHC fue de 41.36 $\pm 1.81 \mathrm{D}$, mientras que la $\mathrm{Km}$ tomada del Pentacam AXL fue de $41.71 \pm 1.61 \mathrm{D}$. Se analizaron las EKR, que a $3.0 \mathrm{~mm}$ fueron de $41.22 \pm 1.76 \mathrm{D}$, a $4.0 \mathrm{~mm}$ de 41.21 $\pm 1.73 \mathrm{D}$ y a $4.5 \mathrm{~mm}$ de $41.22 \pm 1.71 \mathrm{D}$. El TRCP fue de $41.22 \pm 1.73 \mathrm{D}$ a $4.0 \mathrm{~mm}$, mientras que el True $K$ fue de $41.22 \pm 1.72 \mathrm{D}$. Además, se analizaron las diferencias entre el poder corneal aportado por el topógrafo Pentacam AXL y el obtenido con el MHC, con diferencias significativas en la queratometría media de $0.35 \pm 0.75 \mathrm{D}(p=0.02)$. Las EKR a $3.0,4.0$ y $4.5 \mathrm{~mm}$ no tuvieron variaciones importantes; véase que hubo una diferencia media de $-0.14 \pm 0.71 \mathrm{D}(\mathrm{p}=0.05) \mathrm{a}$ 3.0 y $4.0 \mathrm{~mm}$, y de $-0.13 \pm 0.67 \mathrm{D}(\mathrm{p}=0.05)$ a $4.5 \mathrm{~mm}$. Tampoco hubo cambios significativos en el TRCP a $4.0 \mathrm{~mm}$, encontrándose $-0.13 \pm 0.77 \mathrm{D}(\mathrm{p}=0.09)$, ni en el True $K$, que fue de $-0.14 \pm 0.73 \mathrm{D}(\mathrm{p}=0.06)$.

En la tabla 3 se muestra el poder de la LIO calculado con los diferentes valores de poder corneal obtenidos 
Tabla 2. Poder corneal obtenido por el método de historia clínica y por el topógrafo Pentacam AXL

\begin{tabular}{|c|c|c|c|}
\hline Poder corneal (D) & Media \pm DE & Diferencia \pm DE & $\mathbf{p}^{*}$ \\
\hline $\mathrm{MHC}$ & $41.36 \pm 1.81$ & - & - \\
\hline $\mathrm{Km}$ & $41.71 \pm 1.61$ & $0.35 \pm 0.75$ & 0.02 \\
\hline EKR a $3.0 \mathrm{~mm}$ & $41.22 \pm 1.76$ & $-0.14 \pm 0.71$ & 0.05 \\
\hline EKR a $4.0 \mathrm{~mm}$ & $41.21 \pm 1.73$ & $-0.14 \pm 0.71$ & 0.05 \\
\hline EKR a $4.5 \mathrm{~mm}$ & $41.22 \pm 1.71$ & $-0.13 \pm 0.67$ & 0.05 \\
\hline TRCP a $4.0 \mathrm{~mm}$ & $41.22 \pm 1.73$ & $-0.13 \pm 0.77$ & 0.09 \\
\hline True $K$ & $41.22 \pm 1.72$ & $-0.14 \pm 0.73$ & 0.06 \\
\hline
\end{tabular}

Tabla 3. Poder del lente intraocular calculado con queratometrías obtenidas por el método de historia clínica y por el topógrafo Pentacam AXL

\begin{tabular}{|c|c|c|c|}
\hline Poder corneal (D) & Media $\pm \mathrm{DE}$ & Diferencia \pm DE & $\mathbf{p}^{*}$ \\
\hline $\mathrm{MHC}$ & $21.37 \pm 1.70$ & - & - \\
\hline $\mathrm{Km}$ & $20.11 \pm 2.75$ & $-1.25 \pm 2.57$ & $<0.01$ \\
\hline EKR a $3.0 \mathrm{~mm}$ & $21.56 \pm 1.51$ & $0.19 \pm 0.96$ & 0.05 \\
\hline EKR a $4.0 \mathrm{~mm}$ & $21.56 \pm 1.54$ & $0.19 \pm 0.96$ & 0.05 \\
\hline EKR a $4.5 \mathrm{~mm}$ & $21.55 \pm 1.56$ & $0.18 \pm 0.90$ & 0.05 \\
\hline TRCP a $4.0 \mathrm{~mm}$ & $21.54 \pm 1.66$ & $0.21 \pm 1.01$ & 0.09 \\
\hline True $K$ & $21.52 \pm 1.63$ & $0.15 \pm 1.00$ & 0.14 \\
\hline
\end{tabular}

mediante el topógrafo Pentacam AXL y el MHC, así como la diferencia entre ellos. El poder medio de la LIO calculado mediante MHC fue de $21.37 \pm 1.70 \mathrm{D}$, mientras que por $\mathrm{Km}$ fue de $20.11 \pm 2.75 \mathrm{D}$, con una diferencia significativa de $-1.25 \pm 2.57(p<0.01)$. Por otra parte, los poderes de las lentes mediante el empleo de las EKR a 3.0, 4.0 y $4.5 \mathrm{~mm}$ fueron de 21.56 $\pm 1.51 \mathrm{D}, 21.56 \pm 1.54 \mathrm{D}$ y $21.55 \pm 1.56 \mathrm{D}$, respectivamente, sin diferencias estadísticas $(p=0.05)$. De igual manera, no hubo diferencias significativas $(p>0.05)$ en el poder de la LIO calculado mediante TRCP a $4.0 \mathrm{~mm}(21.54 \pm 1.66 \mathrm{D})$ y con el poder corneal real $(21.52 \pm 1.63 \mathrm{D})$.

\section{Discusión}

Con la introducción y el desarrollo a principios de siglo de la cirugía refractiva en Cuba se han intervenido miles de pacientes, lo que ha permitido establecer criterios propios y normativas. En el presente estudio se incluyeron pacientes con 3 meses como mínimo desde la $C R C$, pues es el momento en que la mayoría de los autores coinciden en que se alcanza la estabilidad refractiva ${ }^{17,19}$. A todos los pacientes se les realizó LASEK, coincidiendo con el estudio de Pérez Range ${ }^{17}$, en el cual hubo un predominio del empleo de LASEK sobre LASIK (Laser Assited in Situ Keratomileusis). En otros estudios, como el de Cabrera, et al. ${ }^{20}$, se prefiere también LASEK empleando mitomicina $\mathrm{C}$, que ha demostrado ser tan confiable como LASIK para la corrección de ametropías. Rojas y González ${ }^{21}$, en una evaluación morfométrica corneal in vivo mediante microscopia confocal, demuestran que LASEK presenta una menor repercusión en la homeostasis corneal en cuanto a menor pérdida de queratocitos, mayor preservación del lecho corneal residual y mejor recuperación del plexo nervioso sub-basal al año de la cirugía. Sin embargo, otros autores abogan por la técnica LASIK ${ }^{22}$. Como ambas ofrecen ventajas y desventajas, que deben ser evaluadas por el cirujano y comunicadas al paciente para arribar a la conducta final, a partir de un acuerdo objetivamente establecido, la diferencia pudiera venir dada porque permiten una calidad visual muy similar, pero LASEK aporta mayor seguridad en cuanto al desarrollo de ectasias posquirúrgicas, ofreciendo córneas más estables, así como una calidad de vida superior cuando se emplea mitomicina $C$ (que previene el haze, opacidad corneal por cicatrización superficial del estroma), pues para los pacientes es muy importante reconocerse como individuos totalmente recuperados, sin limitaciones para incorporarse a sus labores sociales, ya que no tienen que preocuparse por el cuidado del colgajo corneal, como ocurre con LASIK.

Hubo predominio de los pacientes de sexo femenino. En el estudio realizado por Kuo, et al. ${ }^{23}$ con el objetivo de identificar las características de los pacientes que solicitan CRC, los investigadores reportaron que este procedimiento lo buscan sobre todo los jóvenes y las mujeres, en concordancia con las investigaciones de Cumberland, et al. ${ }^{24}$ en el Reino Unido y de Hashemi, et al. ${ }^{25}$ en Irán. Este fenómeno puede ser explicado en parte por el mayor deseo estético en las mujeres, y 
puede ser una razón que explique su predominio en el estudio.

En cuanto a la edad y el error refractivo, los resultados de este trabajo están en correspondencia con el protocolo para cirugía refractiva vigente en el ICORPF, ya que es para mayores de 21 años con estabilidad de la refracción y sin límite superior de edad, mientras mantengan el cristalino transparente y cumplan con los criterios para cirugía refractiva con láser ${ }^{26}$.

El proceso de cálculo de la potencia de la LIO debe modificarse cuando se practica en un ojo con CRC previa, ya que existen dos fuentes de error: la incorrecta predicción de la ELP por parte de la fórmula y la determinación errónea de la potencia de la córnea por parte de la queratometría; la corrección de estos dos factores permitirá realizar un cálculo correcto en estos ojos. La mayoría de las fórmulas para el cálculo de la LIO se basan en la posición de la lente con respecto a la córnea, es decir, en la ELP, para aumentar su exactitud ${ }^{1-4}$.

La potencia dióptrica total de la córnea es la suma del poder de la cara anterior (lente convexa) y de la posterior (lente cóncava). Tras la cirugía fotoablativa se produce un cambio en la curvatura de la superficie anterior (se aplana en el caso de la cirugía por miopía y se curva tras la cirugía por hipermetropía), mientras que no cambia la curvatura corneal posterior (o en todo caso el cambio es mínimo). Esta alteración de la relación en la que se basa el valor del índice queratométrico estándar (1.3375) conduce a una sobrestimación de la potencia corneal total por parte del queratómetro. Otros factores implicados en este error son la mayor esfericidad central en la córnea, el cambio en el índice de refracción del estroma corneal y la medición más periférica, ya que las miras se proyectan más periféricas en una córnea aplanada. La queratometría tradicional y la queratometría simulada por la topografía corneal estiman la potencia corneal midiendo los $3,2 \mathrm{~mm}$ centrales de la superficie anterior. Para una córnea normal prolata esta asunción es adecuada, pero tras una cirugía refractiva la relación se altera ${ }^{1-4}$.

En este estudio, la Km fue la que presentó diferencias significativas, lo cual está justificado por ser el poder dióptrico total "estimado» a partir de la cara anterior de la córnea, en una zona de estudio de aproximadamente $3 \mathrm{~mm}$ centrales y con utilización del índice queratométrico de referencia 1.3375; de ahí que los instrumentos que miden tanto la superficie anterior como la posterior, como el Pentacam, pueden disminuir este error en la determinación de la potencia corneal total.
El Pentacam ofrece las EKR, valores que se obtienen en el módulo denominado por Dr. Jack Holladay como Holladay Report, diseñado para tener en cuenta el efecto refractivo (ley de Snell de la refracción) y el efecto de la superficie posterior de la córnea. Con este mapa, el error del índice 1.3375 crea en un mapa sagital que se añade a este compensando las cargas, lo cual puede asemejarlo con el resultado del mapa de TRCP. La TRCP facilita al novedoso Pentacam-AXL los datos totales de la córnea, en una zona de estudio de $4 \mathrm{~mm}$ (zona efectiva pupilar), teniendo en cuenta los índices refractivos reales (aire $=1$, córnea $=1.376$ y medio acuoso $=1.336$ ) y el poder dióptrico total «real» de la córnea, ya que considera condiciones reales ópticas de la córnea, tomando en cuenta la cara anterior y posterior con sus correctos índices refractivos, además de la paquimetría y las aberraciones corneales centrales y periféricas. Tanto las EKR como la TRCP hacen al Pentacam ideal en pacientes con CRC previa ${ }^{3-15}$.

En este trabajo hubo diferencias significativas de la $\mathrm{Km}$ con el poder calculado por el MHC, no así de las EKR a 3.0, 4.0 y $4.5 \mathrm{~mm}$ ni de la TRCP a $4.0 \mathrm{~mm}$ aportadas por el Pentacam-AXL, las cuales resultaron similares, coincidiendo con trabajos anteriores como el de Karunaratne ${ }^{13}$, quien comparó las EKR del Pentacam con la Km. En un estudio, Shajari, et al. ${ }^{10}$ compararon en 93 ojos de 93 pacientes la queratometría total del IOL Master 700 con la K simulada, la TCRP y el true net power del Pentacam-AXL, y concluyeron que la queratometría total del IOL 700 no es intercambiable con la TCRP, aunque esta última es una medida recientemente introducida y por ello son necesarios más estudios, sobre todo por su valor en el cálculo de LIO tóricas.

Las EKR a 3.0, 4.0 y $4.5 \mathrm{~mm}$ calculadas en este estudio mediante el programa Holladay Report no muestran diferencias con la queratometría media calculada por el MHC, de modo similar a otras investigaciones realizadas en el ICORPF. Cuan, et al. ${ }^{14}$ observaron una mayor correlación con la EKR a $3 \mathrm{~mm}$. Es necesario aclarar que en su investigación solo compararon con el MHC y eran pacientes miopes tratados con láser excímer. Cárdenas, et al. ${ }^{27}$ realizaron en igual grupo de pacientes una comparación de los valores aportados por el Pentacam y por el método de Maloney, y obtuvieron las menores diferencias en las EKR a 3.0 y $4.0 \mathrm{~mm}$. En cambio, Pérez, et al. ${ }^{28}$, en un estudio con el objetivo de correlacionar los valores queratométricos obtenidos por el programa Holladay Report del Pentacam y por el método de Maloney, concluyen que todas las variables muestran una correlación positiva 
estadísticamente significativa con los valores obtenidos por el método de Maloney, pero los mayores valores de correlación se correspondieron con las EKR a 4.0 y $4.5 \mathrm{~mm}$, lo cual indicó su mayor aproximación a lo calculado por la fórmula de Maloney. Es importante señalar que este estudio se hizo en ojos operados con queratotomía radial y diagnóstico de catarata.

Viteri ${ }^{29}$ plantea que, según el perfil de ablación, algunos cirujanos prefieren la EKR a 2 o $3 \mathrm{~mm}$, y en su opinión hay que hacer esfuerzos adicionales para determinar la zona óptima de las EKR para casos específicos. En otros estudios, los autores encontraron la mayor similitud a los valores queratométricos del $\mathrm{MHC}$ en el poder corneal aportado por las EKR del programa Holladay Report, dentro de una serie de mediciones queratométricas realizadas por el topógrafo Pentacam en pacientes con CRC con láser excímer ${ }^{30,31}$.

Otro aspecto estudiado fue la diferencia con el True $K$, poder corneal que utiliza el Pentacam-AXL para el cálculo de la lente con la fórmula de Barret True $K$, en cuya determinación se tienen en cuenta los cambios después de la cirugía refractiva para calcular la queratometría preoperatoria y predice la contribución de la córnea posterior. La fórmula matemática del método de Barrett True- $K$ no se ha publicado. Se sabe que el índice queratométrico es modificado y que utiliza las fórmulas Barrett Universal II y la Doble K; se emplea en los pacientes con antecedentes de cirugía refractiva y en ojos de cualquier tamaño. Varios estudios avalan sus resultados refractivos y la consideran una de las mejores opciones en pacientes con antecedentes de cirugía refractiva $^{12,13,16}$. En este trabajo no hubo diferencias significativas con el True $K$.

Wang, et al. ${ }^{32}$ determinaron los valores promedio de la diferencia entre la queratometría total estándar y la determinada por topografía, y observaron que en los ojos miopes, que por lo general tienden a presentar mayor longitud axial, los valores de la diferencia tendieron a ser menores que los observados en los pacientes hipermétropes estudiados por ellos (ojos con menor longitud axial).

En cuanto al cálculo de la LIO por los distintos métodos, en la presente investigación no se encontraron diferencias significativas entre la utilización del poder corneal calculado con el MHC y las diferentes mediciones aportadas por el Pentacam (EKR a 3.0, 4.0 y $4.5 \mathrm{~mm}$, TRCP y True $\mathrm{K}$ ); sin embargo, sí hubo una diferencia significativa del poder de la LIO calculado con la $\mathrm{Km}$, no solo estadísticamente, sino también desde el punto de vista clínico, donde una dioptría de error de la lente puede equivaler a tres dioptrías en la refracción.

En un trabajo realizado por Pérez, et al. ${ }^{28}$ con el objetivo de comparar los resultados visuales de los pacientes con CRC, operados de catarata e implante de LIO, calculados por el método de Maloney y por Pentacam, se halló que tanto los pacientes en quienes se empleó el método de Maloney como aquellos en los que se utilizó el Pentacam para obtener el poder corneal para el cálculo de la LIO los resultados visuales fueron favorables; sin embargo, es importante señalar que se alcanzó la emetropía en un porciento mayor en el grupo en que se utilizó la EKR (50\%) con respecto al que utilizó la $\mathrm{K}$ calculada por Maloney (43.48\%), por lo que se concluye que ambos métodos brindan resultados visuales favorables, pero el cálculo del poder dióptrico de la LIO a partir de las EKR aportadas por el Holladay Report del topógrafo Pentacam ofrece mejores resultados visuales para los pacientes con antecedentes de CRC. De igual manera, otros trabajos han concluido que el topógrafo Pentacam, mediante el programa Holladay Report, aporta poderes corneales que pueden ser utilizados para el cálculo de la LIO en miopes o hipermétropes con cirugía refractiva con láser previa $^{12,17}$.

La fórmula Barrett True $K$ resulta útil para el cálculo de la LIO en pacientes con CRC previa. La obtención de resultados refractivos precisos hace que en la actualidad sea una de las mejores opciones en estos pacientes ${ }^{12,13}$. Por otra parte, en el ICORPF, la fórmula Barret True $K$ incorporada al Pentacam-AXL resultó útil para el cálculo de la LIO en pacientes con catarata y CRC previa ${ }^{16}$.

\section{Conclusiones}

El Pentacam, mediante el Programa Holladay Report, aporta poderes corneales que pueden ser utilizados para el cálculo de la LIO en ojos miopes que tengan cirugía previa con láser excímer, ya que las EKR que aporta el Holladay Report del Pentacam, así como la TRCP y el True $K$ utilizado por la fórmula de Barret True $K$, no difieren del poder corneal obtenido por el MHC en ojos con cirugía refractiva con láser previa para corregir la miopía; no así la Km, que sí difiere.

\section{Financiación}

Los autores declaran que no se recibió financiación para llevar a cabo esta investigación. 


\section{Conflicto de intereses}

Los autores no tienen ningún conflicto de intereses para reportar.

\section{Responsabilidades éticas}

Protección de personas y animales. Los autores declaran que para esta investigación no se han realizado experimentos en seres humanos ni en animales.

Confidencialidad de los datos. Los autores declaran que han seguido los protocolos de su centro de trabajo sobre la publicación de datos de pacientes.

Derecho a la privacidad y consentimiento informado. Los autores han obtenido el consentimiento informado de los pacientes y/o sujetos referidos en el artículo. Este documento obra en poder del autor de correspondencia.

\section{Bibliografía}

1. Galvis V, Tello A, Jaramillo LC, Castillo AM, Pareja LA, Camacho PA Cambios corneales producidos por la cirugía refractiva con excimer láser: revisión de tema. MÉD UIS. 2017;30:99-105.

2. Savini G, Hoffer KJ. Intraocular lens power calculation in eyes with previous corneal refractive surgery. Eye Vis (Lond). 2018;5:18-27.

3. Cárdenas T. Poder corneal postcirugía fotoablativa para el cálculo del lente intraocular. [Tesis de Doctorado.] La Habana: Universidad de Ciencias Médicas de La Habana; 2015.

4. Cárdenas T, Guerra M, Hernández I, Cruz D, Montero E, Hernández R. Principios para el cálculo de la lente intraocular tras cirugía refractiva corneal. Rev Cub Oftalmol. 2016;29(1).

5. Cho K, Lim DH, Yang C, Chung ES, Chung TY. Comparison of intraocular lens power calculation methods following myopic laser refractive surgery: new options using a rotating Scheimpflug camera. J Ophthalmol. 2018:32:497-505.

6. Kang BS, Han JM, Oh JY, Kim MK, Wee WR. Intraocular lens power calculation after refractive surgery: a comparative analysis of accuracy and predictability. Korean J Ophthalmol. 2017:31:479-88.

7. Cho K, Lim DH, Yoo YS, Chung TY. New method for intraocular lens power calculation using a rotating Scheimpflug camera in eyes with corneal refractive surgery. BMC Ophthalmol. 2020;10:8992.

8. Pereira JMM, Neves A, Alfaiate $P$, Santos M, Aragao H, Sousa JC. Lenstar ${ }^{\circledR}$ LS 900 vs Pentacam®-AXL: comparative study of ocular biometric measurements and intraocular lens power calculation. Eur J Ophthalmol. 2018;28:645-51.

9. Haddad JS, Barnwell E, Rocha KM, Ambrosio R Jr, Waring Iv GO. Comparison of biometry measurements using standard partial coherence interferometry versus new Scheimpflug tomography with integrated axial length capability. J Clin Ophthalmol. 2020;14:353-8.

10. Shajari M, Sonntag R, Ramsauer M, Kreutzer T, Vounotrypidis E, Kohnen $T$, et al. Evaluation of total corneal power measurements with a new optical biometer. J Cataract Refract Surg. 2020;46:675-81.
11. Abulafia A, Hill WE, Koch DD, Wang L, Barrett GD. Accuracy of the Barrett True-K formula for intraocular lens power prediction after laser in situ keratomileusis or photorefractive keratectomy for myopia. J Cataract Refract Surg. 2016;42:363-9.

12. Zhu W, Zhang FJ, Li Y, Song YZ. Stability of the Barrett True-K formula for intraocular lens power calculation after SMILE in Chinese myopic eyes. Int J Ophthalmol. 2020;13:560-6.

13. Karunaratne $\mathrm{N}$. Comparison of the Pentacam equivalent keratometry reading and $\mathrm{IOL}$ Master keratometry measurement in intraocular power calculations. Clin Exp Ophthalmol. 2013:41:825-34.

14. Cuan Y, Pérez E, Montero E, Santiesteban I, Ortega L, Cárdenas T. Utilidad del topógrafo Pentacam para medir el poder corneal después de cirugía refractiva con excímer láser. Rev Cub Oftalmol. 2010;23 (Supl 1):513-21.

15. Cárdenas T, Guerra M, Pérez EC, Cuan $Y$, Hernández I. Cirugía refractiva láser previa y cálculo del lente intraocular en miopes. Rev Cub Oftalmol. 2017;30(2).

16. Cangrejo Forero El. Resultados del cálculo de lente en operados de catarata y cirugía refractiva: fórmula Barrett True K. [Tesis.] La Habana: Universidad de Ciencias Médicas de La Habana; 2020.

17. Pérez Rangel Y. Cálculo del lente intraocular en pacientes con cirugía refractiva láser previa. [Tesis.] La Habana: Universidad de Ciencias Médicas de La Habana; 2019.

18. Di Ruggiero M. Declaración de Helsinki, principios y valores bioéticos en juego en la investigación médica con seres humanos. Revista Colombiana de Bioética. 2011;6:125-44

19. Rey DV, Moreno Montoya J. Resultado visual a los 3 meses de cirugía LASEK. Rev Mex Oftalm. 2017:91:247-53.

20. Cabrera Martínez A, Cabrera Martínez JA, Tirado Martínez OM. Resultados refractivos en pacientes operados por LASIK versus LASEK con mitomicina C. Rev Cub Oftalmol. 2009;36(7).

21. Rojas Álvarez E, González Sotero J. LASIK vs. LASEK desde la perspectiva morfométrica corneal in vivo. Rev Mex Oftalm. 2013;87:145-57.

22. Sierra PB, Hardten DC. LASIK. En: Yanoff M, Duker JS, editores. Oftalmología. 5. a ed. Barcelona: Elsevier; 2019. p. 95-105.

23. Kuo I, Lee B, Wang J. Outcomes of refractive surgery consultations at an academic center: characteristics associated with proceeding (or not proceeding) with surgery. J Ophthalmol. 2020;2020:4354085.

24. Cumberland PM, Chianca A, Rahi JS. Laser refractive surgery in the UK Biobank study: frequency, distribution by sociodemographic factors, and general health, happiness, and social participation outcomes. J Cataract Refract Surg. 2015;41:2466-7.

25. Hashemi H, Khabazkhoob M, Pakzad R, Yekta A, Nojomi M, Nabovati P. The characteristics of excimer laser refractive surgery candidates. Eyes Contact Lens. 2018;44 (Suppl 1):S158-62.

26. Cárdenas T, Machado E, Guerra M. Cirugía refractiva corneal. En: Ríos M, Fernández L, Hernández JR, Ramos M, editores. Oftalmología. Diagnóstico y tratamiento. La Habana: Ciencias Médicas; 2018. p. 89-93.

27. Cárdenas T, Ravelo W, Capote A, Pérez EC, Cuan Y, Hernández I. Poder corneal postcirugía fotoablativa en miopes. Sistema Scheimpflug topógrafo Pentacam vs método de Maloney. Rev Cub Oftalmol. 2014;27(1)

28. Pérez EC, Cárdenas T, Hormigó I, Alarcón E, Trujillo K, Santiesteban I. Correlación de los valores queratométricos del Pentacam y el método de Maloney. Rev Cub Oftalmol. 2016;29(3)

29. Viteri E. Uso del topógrafo Pentacam para calcular el poder de la LIO. High Lights of Ohthalmology. 2008;36(3).

30. Savini G, Barboni P, Profazio V, Zanini M, Hoffer KJ. Corneal power measurements with the topographer Pentacam Scheimpflug camera after myopic excimer laser surgery. J Cataract Refract Surg. 2008;34:809-13

31. Jain R, Dilraj G, Grewal SPS. Repeatability of corneal parameters with topographer Pentacam after laser in situ keratomileusis. Indian J. Ophthalmol. 2007:55:341-7.

32. Wang L, Spektor T, de Souza RG, Koch DD. Evaluation of total keratometry and its accuracy for intraocular lens power calculation in eyes after corneal refractive surgery. J Cataract Refract Surg. 2019;45:1416-21. 